
\title{
25 Research Square \\ Gut Microbiota Profiles of Systemic Lupus Erythematosus Patients with Anxiety or Depression
}

alvina widhani ( $\nabla$ alvina.widhani@gmail.com )

Universitas Indonesia https://orcid.org/0000-0002-1848-6227

Meutia Gebrina

Universitas Indonesia Fakultas Kedokteran

Rudi Putranto

Universitas Indonesia Fakultas Kedokteran

Murdani Abdullah

Universitas Indonesia Fakultas Kedokteran

Ikhwan Rinaldi

Universitas Indonesia Fakultas Kedokteran

Samsuridjal Djauzi

Universitas Indonesia Fakultas Kedokteran

Fransiscus D. Suyatna

Universitas Indonesia Fakultas Kedokteran

Beti Ernawati Dewi

Universitas Indonesia Fakultas Kedokteran

Andi Yasmon

Universitas Indonesia Fakultas Kedokteran

Susan Rahayu

Universitas Indonesia Fakultas Kedokteran

\section{Research}

Keywords: anxiety, depression, gut microbiota, systemic lupus erythematosus

Posted Date: September 16th, 2021

DOI: https://doi.org/10.21203/rs.3.rs-885639/v1

License: (a) (1) This work is licensed under a Creative Commons Attribution 4.0 International License.

Read Full License 


\section{Abstract \\ Background}

Patients with systemic lupus erythematosus (SLE) often experience anxiety and depression. Recent studies have shown involvement of intestinal dysbiosis in SLE and also psychosomatic disorders. However, there are no reports on the gut microbiota profile of patient with both conditions: SLE and anxiety or depression. We aimed to study gut microbiota profiles among SLE patients with gastrointestinal symptoms and anxiety or depression by sequencing V3-V4 region of the 16S rRNA gene from the stool samples.

\section{Results}

Of the 41 SLE patients who participated in the study, $53.66 \%$ had anxiety and $14.63 \%$ had depression. We found a higher proportion of Bacteroidetes and lower diversity indices in patients with anxiety than in those without anxiety. We also found a higher proportion of Bacteroidetes and lower Firmicutes/Bacteroidetes ratios and diversity indices in patients with depression than in those without depression. Moreover, compared to other groups, patients with symptoms of both anxiety and depression had the highest proportion of Bacteroidetes and lowest proportion of Firmicutes, Firmicutes/Bacteroidetes ratios, and diversity indices. Further analysis showed that there was a significant correlation between the proportion of Bacteroides and the anxiety score $(r=0.349 ; p=0.03)$ as well as with lupus activity $(r=0.36 ; p=0.02)$. There was also significant correlation between diversity indices and lupus activity $(r=-0.34 ; p=0.03$ for Chao1 index, $r=-0.38 ; p=0.01$ for Shannon index, and $r=$ $-0.33 ; p=0.03$ for richness index).

\section{Conclusions}

SLE patients with both anxiety and depression showed more unfavorable gut dysbiosis parameter compared to SLE patients with only anxiety or depression and SLE patients without anxiety or depression. There was positive correlation between proportion of Bacteroides and lupus disease activity and negative correlation between diversity indices and lupus disease activity.

\section{Background}

Systemic lupus erythematosus (SLE) is a chronic systemic autoimmune disease with unpredictable progression in which organs and cells undergo damage initially mediated by tissue-binding autoantibodies and immune complexes. ${ }^{1,2,3}$ Lupus disease activity and severity, age, sex, social and culture problem can lead to psychosomatic problems that complicate patient management. ${ }^{4}$ A metaanalysis by Zhang et al showed that the prevalence of depression and anxiety in SLE was $30 \%$ and $40 \%$, 
respectively, both of which were assessed using the Hospital Anxiety and Depression Scale (HADS) questionnaire. $^{5}$

SLE and psychosomatic disorders are linked by several factors: autoantibodies to nerve cell antigens, ${ }^{6}$ female hormonal factors, ${ }^{7,8}$ and gut dysbiosis. ${ }^{9,10}$ Gut dysbiosis results in the activation of the immune response that causes inflammatory reactions in intestinal lymphoid tissue ${ }^{11}$ and reduction in levels of short-chain fatty acids (SCFAs), which are sources of nutrition for the epithelium. ${ }^{12}$ This condition disrupts the integrity of the intestinal epithelium, which can lead to systemic inflammation. ${ }^{13}$

Systemic inflammation affects neurotransmitter metabolism, causing serotonin deficiency. ${ }^{14}$ High levels of cortisol in inflammation increase both brain glutamate levels, causing overexcitation, and free radicals, which impair neuronal activity, causing decreased gamma aminobutyric acid (GABA) production. ${ }^{15}$ In addition, beneficial microbes-whose levels decrease in inflammation-are a source of neurotransmitters such as serotonin and GABA. Serotonin deficiency plays an important role in the pathogenesis of depression, whereas GABA deficiency plays an important role in anxiety. ${ }^{16}$

To date, there have been no published studies on gut microbiota profiles among SLE patients with anxiety or depression. Hence, we aimed to determine the gut microbiota profiles of patients with SLE who had gastrointestinal symptoms and anxiety or depression.

\section{Methods}

The study was conducted at the outpatient clinic of Cipto Mangunkusumo Hospital, Jakarta, Indonesia. The inclusion criteria were patients with SLE according to the American College of Rheumatology 1997 criteria, age 18-60 years, and gastrointestinal symptoms (abdominal pain, bloating, diarrhea, or constipation). Subjects who were pregnant or breastfeeding, experiencing acute infections, receiving antibiotic therapy, taking yogurt or supplements containing probiotics/prebiotics/synbiotics in the last three weeks, receiving corticosteroid therapy equivalent to prednisone more than $20 \mathrm{mg} /$ day, and not willing to participate were excluded. This study was approved by the Research Ethics Committee of the Faculty of Medicine, University of Indonesia (registration ID: 804/UN2.F1/ETIK/2017). Written informed consent was obtained from all participants. Demographical data, SLE disease activity score (Systemic Lupus Erythematosus Disease Activity Index 2000, SLEDAI-2K) and medication information were collected.

Symptoms of anxiety and depression were assessed using the Hospital Anxiety and Depression Scale (HADS) questionnaire. Anxiety or depression was defined as an HADS score more than 7 for the related domain. Patients were assisted in completing the questionnaire. Dietary assessment was performed by a certified nutritionist using a food recall questionnaire.

Patients received explanation and written instructions on how to collect stool samples. Stool nucleic acid collection tubes (Norgen, Biotec Corp, Thorold, ON, Canada) were used. Fecal samples were stored at - 
$80^{\circ} \mathrm{C}$ until DNA extraction. DNA extraction was done using QIAamp DNA stool mini kits (QIAGEN, Hilden, Germany) according to the manufacturer's instructions. DNA purity and concentration were measured using a NanoDrop 2000 Spectrophotometer (Thermo Fisher Scientific, Waltham, MA, USA) and a Qubit Fluorometer (Invitrogen Life Technologies, Carlsbad, CA, USA). Before amplification, DNA with a purity ratio of 1.8 to 2.0 was diluted to a concentration of $5 \mathrm{ng} / \mu \mathrm{L}$. Polymerase chain reaction was performed using forward and reverse primers specific for $16 \mathrm{~S}$ rRNA. The amplicons were purified by standard procedures, quantified, pooled, and sequenced using MiSeq Reagent Kits (Illumina, San Diego, CA, USA) according to the manufacturer's instructions.

After sequencing, the reads were assembled using FLASH (v1.2.7, http://ccb.jhu.edu/software/FLASH/), and the data were analyzed on the USEARCH pipeline (https://www.drive5.com/usearch/) using default parameters. Unique reads and their abundance values were generated using the 'fastx_uniques' command in USEARCH. Operational taxonomic unit (OTU) clustering and chimera removal were performed using the UPARSE algorithm to produce OTUs with $>97 \%$ similarity. Taxonomic affiliation of each OTU was predicted with USEARCH against the Ribosomal Database Project Training Set v.16. Alpha diversity (richness, Chao1, and Shannon) indices were calculated in USEARCH using OTU tables normalized to 10,000 reads.

Other statistical analyses were performed using IBM SPSS statistics (version 20.0.0; IBM Corp., Armonk, NY, USA). Data were expressed as mean \pm standard deviation (for normally distributed data) and median (interquartile range for data with skewed distributions). Comparisons of numeric data between the two groups were analyzed using independent $t$-tests if the data were normally distributed. Otherwise, the comparisons were analyzed using the non-parametric Mann-Whitney $U$ test. P-values $<0.05$ were considered statistically significant.

\section{Results}

All subjects were women $(n=41)$ with median age was 33 years. Table 1 shows the characteristics of the subjects. The median body mass index, SLEDAI-2K score, and steroid dose were $21.6 \mathrm{~kg} / \mathrm{m}^{2}, 12$, and 5 $\mathrm{mg}$ equivalent to prednisone per day, respectively. The proportions of patients with anxiety and depression in this study were $53.66 \%$ and $14.63 \%$, respectively with $12.2 \%$ of the subjects had both anxiety and depression. About $43.9 \%$ subjects did not experience anxiety or depression. 
Table 1

Patient characteristics

\section{Characteristics}

Age (years), median (min-max)

Body mass index $(\mathrm{BMI})\left(\mathrm{kg} / \mathrm{m}^{2}\right)$, median (min-max)

SLEDAl-2k, median (min-max)

Steroid dose ( $\mathrm{mg} /$ day equivalent to prednisone), median (min-max)

Steroid sparing agents, $\mathrm{n}(\%)$

- Hydroxychloroquine

- Mycophenolate mofetil

- Azathioprine

- Cyclosporine

Other medications, $\mathrm{n}(\%)$

- Proton pump inhibitor

- Statin

Food intake (g/day)

- Carbohydrate, mean (SD)

- Fat, median (min-max)

- Protein, mean (SD)

- Fiber, median (min-max)

HADS-A, mean (SD)

Anxiety (HADS-A > 7), n (\%)

HADS-D, median (min-max)

Depression (HADS-D > 7), n (\%) $\mathrm{n}=41$

$33(19-47)$

$21.6(12.99-41.08)$

$12(4-32)$

$5(0-20)$

2 (4.9)

27 (65.9)

$6(14.6)$

$2(4.9)$

20 (48.8)

$2(4.9)$

$185.5(64.27)$

$40(18.58-83.52)$

47.46 (21.84)

$9.52(1.75-38.8)$

$7.88(2.82)$

$22(53.66)$

$5(0-14)$

$6(14.63)$

$\mathrm{BMI}=$ body mass index, SLEDAI-2k = systemic lupus erythematosus disease activity index 2000; SD = standard deviation; HADS-A = Hospital Anxiety and Depression Scale-Anxiety, HADS-D = Hospital Anxiety and Depression Scale-Depression

The characteristics and gut microbiota comparison between SLE patients with and without anxiety are shown in Table 2. The median SLEDAl-2k scores were 14 and 10 in the anxiety and non-anxiety groups, respectively. The proportion of Bacteroidetes was higher and the diversity indices were lower in SLE patients with anxiety than in patients without anxiety. The characteristics and gut microbiota comparison 
between SLE patients with and without depression are also shown in Table 2. Among patients with depression, the use of proton pump inhibitors was higher (83.33\%) than in the patients without depression (42.86\%). Food intake was lower in patients with depression than in those without depression. Among SLE patients with depression, the proportion of Bacteroidetes was higher, whereas the proportion of Firmicutes, the Firmicutes/Bacteroidetes ratio, and the diversity indices were lower than in patients without depression. The most severe gut dysbiosis was seen in the group that experienced both anxiety and depression: the highest proportion of Bacteroidetes and the lowest proportion of Firmicutes, Firmicutes/Bacteroidetes ratio, and diversity indices (Table 3). 
Table 2

Characteristics and gut microbiota profiles of SLE patients with anxiety and/or depression

\section{Characteristics}

Anxiety

Yes $(n=22) \quad$ No $(n=19) \quad$ Yes $(n=6) \quad$ No $(n=$
Age (years), mean (SD)

$\operatorname{BMI}\left(\mathrm{kg} / \mathrm{m}^{2}\right)$, median (min-max)

Coexistence of depression and anxiety, $\mathrm{n}$

$(\%)$

SLEDAl-2k, median (min-max)

Steroid use (mg/day equivalent to

prednisone), median (min-max)

Concomitant drug use

- PPI, n (\%)

- Statin, n (\%)

Food intake (g/day)

- Fat, mean (SD)

- Protein, mean (SD)

Fiber, median (min-max)

\section{- Carbohydrate, mean (SD)}

30.14

(7.978)

21.43

(12.99-

28.35)

$5(22.73 \%)$

$14(4-32)$

$5(0-20)$

$10(45.45 \%)$

10

(52.63\%)

$1(4.55 \%)$

$1(5.26 \%)$

$0(0 \%)$

$30(24-$

47)

23.64

(9.58)

41.1)

$1(5.26 \%)$

$10(6-22)$

$5(0-20)$

$5.5(5-10)$

$5(83.33 \%)$

$12(6-18)$
$33(19-$

45)

22.16

(3.92)
(48.57\%)

17

$12(4-32)$

$5(0-20)$

17

$2(5.71 \%)$

15

(42.86\%)

2 (5.71\%)
169.2

(65.21)

38.78

(14.05)

42.58

(21.76)

$8.38(1.75-$

38.8)
204.37

(59.3)

48.43

(15.51)

53.12

(21.09)

10.49

$(2.59-37)$
158.44

(81.43)

31.48

(9.09)

33.6

(15.88)

6.48

(1.75-

10.78)
190.14

(61.1)

45.27

(15.38)

49.84

(22.01)

9.85

(2.59-

38.8)

\section{Gut microbiota}

Bacteroidetes proportion (\%), mean (SD)

53.21

(12.59)

51.56

(13.52)

53.07

(8.08)

52.34

33.68

(15.29)

32.21

(32.7)

30.32

(11.88)

Firmicutes proportion (\%), mean (SD)

(32.7)

$(11.88)$

33.46
$(14.34)$

SD, standard deviation; BMI, body mass index; SLEDAI-2k systemic lupus erythematosus disease activity index 2000; PPI, proton pump inhibitor. 


\begin{tabular}{|c|c|c|c|c|}
\hline \multirow[t]{2}{*}{ Characteristics } & \multicolumn{2}{|l|}{ Anxiety } & \multicolumn{2}{|l|}{ Depression } \\
\hline & Yes $(n=22)$ & No $(n=19)$ & Yes $(n=6)$ & $\begin{array}{l}\text { No }(n= \\
35)\end{array}$ \\
\hline $\begin{array}{l}\text { Actinobacteria proportion (\%), median } \\
(\min -\max )\end{array}$ & $\begin{array}{l}0.5(0.03- \\
9.1)\end{array}$ & $\begin{array}{l}0.74 \\
(0.05-2.3)\end{array}$ & $\begin{array}{l}0.76 \\
(0.48- \\
2.31)\end{array}$ & $\begin{array}{l}0.53 \\
(0.03- \\
9.1)\end{array}$ \\
\hline $\begin{array}{l}\text { Verrucomicrobia proportion (\%), median } \\
\text { (min-max) }\end{array}$ & $0(0-2.67)$ & $0(0-1.23)$ & $0(0-0.02)$ & $\begin{array}{l}0(0- \\
2.67)\end{array}$ \\
\hline $\begin{array}{l}\text { Proteobacteria proportion (\%), median } \\
\text { (min-max) }\end{array}$ & $\begin{array}{l}6.18(1.83- \\
40.5)\end{array}$ & $\begin{array}{l}9.02 \\
(4.39- \\
37.3)\end{array}$ & $\begin{array}{l}7.16 \\
(1.83- \\
40.5)\end{array}$ & $\begin{array}{l}7.83 \\
(2.03- \\
37.3)\end{array}$ \\
\hline $\begin{array}{l}\text { Fusobacteria proportion (\%), median (min- } \\
\text { max) }\end{array}$ & $\begin{array}{l}0.04(0- \\
26.7)\end{array}$ & $\begin{array}{l}0.06(0- \\
3.21)\end{array}$ & $\begin{array}{l}0.15(0- \\
0.71)\end{array}$ & $\begin{array}{l}0.04(0- \\
26.7)\end{array}$ \\
\hline $\begin{array}{l}\text { Firmicutes/Bacteroidetes ratio, median } \\
\text { (min-max) }\end{array}$ & $\begin{array}{l}0.57(0.18- \\
3.6)\end{array}$ & $\begin{array}{l}0.55 \\
(0.22-1.6)\end{array}$ & $0.58(0.27)$ & $\begin{array}{l}0.77 \\
(0.65)\end{array}$ \\
\hline \multicolumn{5}{|l|}{ Diversity index } \\
\hline - Richness index, mean (SD) & $\begin{array}{l}846.45 \\
(150.7)\end{array}$ & $\begin{array}{l}873.26 \\
(112.35)\end{array}$ & $\begin{array}{l}783 \\
(187.32)\end{array}$ & $\begin{array}{l}871.9 \\
(120.73)\end{array}$ \\
\hline - Shannon index, mean (SD) & $4.36(0.45)$ & $4.48(0.39)$ & $4.25(0.5)$ & $4.45(0.4)$ \\
\hline - Chao1 index, mean (SD) & $\begin{array}{l}852.45 \\
(151.7)\end{array}$ & $\begin{array}{l}879.77 \\
(113.2)\end{array}$ & $\begin{array}{l}787.55 \\
(187.92)\end{array}$ & $\begin{array}{l}878.41 \\
(121.6)\end{array}$ \\
\hline
\end{tabular}


Table 3

Comparison of gut microbiota between SLE patients with anxiety and depression, anxiety or depression, and no anxiety or depression

\begin{tabular}{|lllll|}
\hline Gut microbiota profile & $\begin{array}{l}\text { Anxiety and } \\
\text { depression }(\mathbf{n}=\mathbf{5})\end{array}$ & $\begin{array}{l}\text { Anxiety only } \\
(\mathbf{n = 1 7})\end{array}$ & $\begin{array}{l}\text { Depression } \\
\text { Only } \\
(\mathbf{n}=1)\end{array}$ & $\begin{array}{l}\text { No anxiety or } \\
\text { depression }(\mathbf{n}=18)\end{array}$ \\
\hline $\begin{array}{l}\text { Firmicutes proportion (\%), } \\
\text { mean (SD) }\end{array}$ & $27.04(9.8)$ & $\begin{array}{l}35.63 \\
(16.28)\end{array}$ & 46.7 & $31.4(12.35)$ \\
\hline $\begin{array}{l}\text { Bacteroidetes proportion } \\
\text { (\%), mean (SD) }\end{array}$ & $54.6(8)$ & $52.8(13.83)$ & 45.4 & $51.9(13.83)$ \\
\hline $\begin{array}{l}\text { Firmicutes/Bacteroidetes } \\
\text { ratio, mean (SD) }\end{array}$ & $0.49(0.17)$ & $0.85(0.83)$ & 1.03 & $0.7(0.45)$ \\
\hline $\begin{array}{l}\text { Diversity index, mean (SD) } \\
\text { - Richness index }\end{array}$ & $780.8(209.34)$ & $\begin{array}{l}865.76 \\
(130.8)\end{array}$ & 794 & $877.67(113.91)$ \\
\hline - Shannon index & $4.17(0.51)$ & $4.42(0.42)$ & 4.67 & $4.47(0.4)$ \\
\hline - Chao1 index & $785.36(210.01)$ & $\begin{array}{l}872.19 \\
(131.76)\end{array}$ & 798.5 & $884.29(114.7)$ \\
\hline SD = standard deviation & & & & \\
\hline
\end{tabular}

Further analysis showed that the disease duration was significantly correlated with the depression score $(r=-0.426, p=0.01)$ (Fig. 1). Analysis at the genus level showed that the proportion of Bacteroides was significantly higher in SLE patients with anxiety than in those without anxiety $(33.45 \%$ vs. $9.78 \% ; p=0.02)$ (Fig. 2a). There was also a significant correlation between the proportion of Bacteroides (genus level) and the anxiety score $(r=0.349, p=0.03)(F i g .2 b)$. The proportion of Bacteroides also had a significant correlation with SLE disease activity $(r=0.36, p=0.02)$ (Fig. 2c). Diversity indices (Chao1, Shannon, and Richness index) also showed significant correlation with SLE disease activity $(r=-0.34, p=0.03 ; r=-0.38$, $p=0.01 ; r=-0.33, p=0.03$, respectively) (Fig. 3a-c).

\section{Discussion}

In our study, the median SLE disease activity score among patients with anxiety was higher than in patients without anxiety. High disease activity might be a physical stressor for patients with SLE, which can cause anxiety. ${ }^{17}$ In addition, we found lower food intake among patients with anxiety, which seems to contradict the theory of emotional eating in anxiety. This difference might be explained by the higher comorbidity of depression in the anxiety group than in patients without anxiety. Depression can cause anhedonia, which results in decreased food intake. ${ }^{18}$

We found a negative correlation between the disease duration and the depression score. This may be explained by Kübler-Ross' theory, which states that there are five stages of grieving: denial, anger, 
bargaining, depression, and acceptance. ${ }^{19}$ Thus, it is likely that patients with longer disease duration had reached the stage of acceptance.

SLE has a two-way relationship with gut dysbiosis. Gut dysbiosis can worsen the pathology of autoimmunity, and SLE can change gut microbiome patterns (dysbiosis). Pro-inflammatory conditions in SLE can also disrupt intestinal integrity. In dysbiosis, which is caused by various factors such as infection, drugs, and dietary patterns, pathogens are more dominant than normal intestinal flora and activate intestinal lymphoid tissue. This response results in the overactivation of lymphocytes and the transregulation of regulatory T cells to Th17 cells. Interleukin-17 (IL-17) released by Th17 cells amplifies the immune response and activates $\mathrm{B}$ cells to produce autoantibodies. ${ }^{13}$ Thus, intestinal dysbiosis can cause loss of self-tolerance in SLE in the preclinical stage.

A study by Hevia et al. had shown that SLE patients have lower Firmicutes/Bacteroidetes ratios (median ratio 1.97) than healthy subjects (median ratio 4.86). ${ }^{20} \mathrm{~A}$ low Firmicutes/Bacteroidetes ratio is one of the parameters of gut dysbiosis. ${ }^{11}$ The median Firmicutes/Bacteroidetes ratio in our subjects was 0.56 (min-max 0.18-3.6). In this study, we also found a significant correlation between some gut microbiota parameters (Bacteroides proportion and diversity indices) and disease activity. A higher proportion of Bacteroides and lower diversity of gut microbiota correlated with higher SLE disease activity.

Pathobionts are potentially pathogenic microbes that can release toxins. ${ }^{21}$ The predominant phylum in the gut is Bacteroidetes, a pathobiont. ${ }^{12}$ Bacteroidetes are microbes that tend to be pathogenic and disrupt the integrity of the intestinal epithelium to cause inflammation. ${ }^{22}$ These conditions can result in changes in synapse architecture, synapse deficits, immune system defects, and progressive inflammation. ${ }^{23}$ In our study, Bacteroidetes counts were found to be higher among SLE patients with anxiety than in those without anxiety. A similar finding was also found in SLE patients with depression. Bacteroides, the main genus in the phylum Bacteroidetes, are pathogenic, highly virulent microbes in the intestine that trigger inflammatory reactions in the intestine. ${ }^{12,24}$

Bacteroides fragilis is a facultative, gram-negative, anaerobic bacterium, which comprises a large proportion of the gut microbiota. It secretes various neurotoxins, including the highly pro-inflammatory $B$. fragilis lipopolysaccharide (BF-LPS). ${ }^{23}$ BF-LPS can trigger the production of nuclear factor-kB, which causes the transcription of a group of proinflammatory microRNAs. In addition to Bacteroides in the gastrointestinal tract, Bacteroidetes also play a role in the secretion of glycoproteins, SCFA imbalance, and the production of toxins. ${ }^{25}$

Inflammation causes the conversion of tryptophan-the precursor of serotonin-to kynurenine, which causes serotonin deficiency. The increased levels of kynurenine cause neurotoxicity and neurodegeneration. ${ }^{14}$ In addition, inflammation is associated with high cortisol levels, which can cause neurotoxicity and decrease GABA production. ${ }^{15}$ The deficiency of GABA and serotonin can also occur because GABA-producing microbes and serotonin-producing microbes are suppressed by pathobionts. ${ }^{16}$ 
GABA deficiency plays a role in the pathogenesis of anxiety, ${ }^{16}$ whereas serotonin deficiency plays a role in depression. ${ }^{10}$

Other contributors to dysbiosis are low diversity indices. ${ }^{26}$ In this study, lower diversity indices were found in SLE patients with anxiety than in those without anxiety and in SLE patients with depression than in those without depression. Loss of microbial diversity is the most constant finding of gut dysbiosis, which may affect the function of the microbial community. High biodiversity helps to maintain a stable ecosystem so that the gut becomes more resilient to many insults. ${ }^{27}$

In this study, there was a lower proportion of Firmicutes and Firmicutes/Bacteroidetes ratio among SLE patients with depression. Firmicutes are the main gut microbiota that produce SCFAs ${ }^{12}$ and also produce a number of neurotransmitters. ${ }^{16} \mathrm{~A}$ lower proportion of Firmicutes may cause inflammation and deficiency of many neurotransmitters, which may result in depression. Our finding was consistent with that of a study by Jiang et al., which showed a decrease in Firmicutes levels and an increase in Bacteroidetes levels in subjects with depression. ${ }^{28}$

Patients who experienced both anxiety and depression showed the most severe gut dysbiosis compared to the other groups. In this group, we found the highest proportion of Bacteroidetes and the lowest proportion of Firmicutes, Firmicutes/Bacteroidetes ratio, and diversity indices. Severe dysbiosis can result in a deficiency of neurotransmitters and inflammation, which might worsen psychosomatic disorders. ${ }^{16}$

The gut microbiome-brain axis is a complex multidirectional system involving the gut microbiota, the enteric nervous system, and the brain. Gut dysbiosis can cause psychosomatic disorders, and psychosomatic disorders can cause gut dysbiosis by affecting dietary patterns. ${ }^{8}$ In our study, we found lower food intake (carbohydrate, fat, protein, and fiber) in SLE patients with anxiety than in those without anxiety and also in SLE patients with depression than in patients without depression. Lower carbohydrate and fiber intake are risk factors for intestinal dysbiosis. ${ }^{29}$ In addition, psychosomatic disorders activate the hypotalamus-pituitary-adrenal axis, which can increase the level of cortisol and disrupt the integrity of the intestinal epithelium. ${ }^{8}$

This is the first study to report the gut microbiota profiles of SLE patients with anxiety or depression, which can give new insight in the pathogenesis and management of psychosomatic problems in SLE patients. However, this study also had limitation. The sample size was small. This study only assessed anxiety and depressive symptoms based on a questionnaire, not based on interviews. In addition, psychosomatic disorders have various influencing factors, including educational background, social and economic factors.

\section{Conclusions}

SLE patients with both anxiety and depression showed more unfavorable gut dysbiosis parameter compared to SLE patients with only anxiety or depression and SLE patients without anxiety or

Page $11 / 17$ 
depression. There was positive correlation between proportion of Bacteroides and lupus disease activity and negative correlation between diversity indices and lupus disease activity.

\section{Declarations}

\section{Acknowledgements}

We would like to thank our colleagues, Fitri Hudayani, Ade Rosanti, and Amalia Shabrina for helping with food intake analysis; Nida Makiyah and Indri for helping with the DNA extraction; and Zulfikar Achmad Tandjung and Redi Aditama for helping with the bioinformatics analysis.

\section{Author's Contributions}

AW made substantial contributions to the conceptual design, data collection, and manuscript writing; MG made substantial contributions to the conceptual design and manuscript writing; RP, MA, and IR made substantial comtributions to data interpretation and revisions to the manuscript; SD, FS, and BD made considerable contributions to the conceptual design, data interpretation, and revisions to the manuscript; AY and SR made substantial contributions to the sample collection, microbiome evaluation, and data analysis; all authors gave approval for publication.

\section{Funding}

The publication process was supported by a grant from the University of Indonesia (NKB4443/UN2.RST/HKP.05.00/2020)

\section{Availability of data and materials}

The metagenomics for the microbiome sequencing was uploaded to Sequence Read Archieve (SRA) with accession number PRJNA761343.

\section{Ethics approval and consent to participate}

This study had been approved by the Research Ethics Committee of the Faculty of Medicine, University of Indonesia (registration ID: 804/UN2.F1/ETIK/2017). Written informed consent was obtained from each patient before enrolment in the study.

\section{Consent for publication}

Not applicable.

Competing interests

The authors declare that they have no competing interests 


\section{References}

1. Hahn BH. Systemic lupus erythematosus. In: Jameson JL, Fauci AS, Kasper DL, Hauser SL, Longo DL, Loscalzo J, editors. Harrison's Principals of Internal Medicine. 20th ed.: McGraw-Hill; 2018.

2. Fortuna G, Brennan MT. Systemic lupus erythematosus: epidemiology, pathophysiology, manifestations, and management. Dent Clin North Am. 2013;57(4):631-55. doi:10.1016/j.cden.2013.06.003.

3. Tselios K, Gladman DD, Touma Z, Su J, Anderson N, Urowitz MB. Disease course patterns in systemic lupus erythematosus. Lupus. 2019;28:114-22. doi:10.1177/0961203318817132.

4. Figueiredo-Braga M, Cornaby C, Bernardes M, Figueiredo M, Mesquita CDS, Costa L, et al. Correlation between physical markers and psychiatric health in a Portuguese systemic lupus erythematosus cohort: The role of suffering in chronic autoimmune disease. PLos One. 2018;13(4):e0195579. doi:10.1371/journal.pone.0195579.

5. Zhang L, Fu T, Yin R, Zhang Q, Shen B. Prevalence of depression and anxiety in systemic lupus erythematosus: A systematic review and meta-analysis. BMC Psychiatry. 2017;17(1):70. doi:10.1186/s12888-017-1234-1.

6. Faust TW, Chang EH, Kowal C, Berlin R, Gazaryan IG, Bertini E, et al. Neurotoxic lupus autoantibodies alter brain function through two distinct mechanisms. Proc Natl Acad Sci U S A. 2010;107(43):18569-74; doi:10.1073/pnas.1006980107

7. Grygiel-Górniak B, Puszczewicz MJ. The influence of endogenous and exogenous sex hormones on systemic lupus erythematosus in pre- and postmenopausal women. Prz Menopauzalny. 2014; 13 (4):262-6; doi:10.5114/pm.2014.45003

8. Rogers GB, Keating DJ, Young RL, Wong ML, Licinio J, Wesselingh S. From gut dysbiosis to altered brain function and mental illness: Mechanisms and pathways. Mol Psychiatry. 2016;21(6):738-48. doi:10.1038/mp.2016.50.

9. de Oliveira GLV, Leite AZ, Higuchi BS, Gonzaga MI, Mariano VS. Intestinal dysbiosis and probiotic applications in autoimmune diseases. Immunology. 2017; 152(1):1-12; doi:10.1111/imm.12765

10. Taylor AM, Holscher HD. A review of dietary and microbial connections to depression, anxiety, and stress. Nutr Neurosci. 2018;23(3):237-50. doi:10.1080/1028415X.2018.1493808.

11. Weiss GA, Hennet T. Mechanisms and consequences of intestinal dysbiosis. Cell Mol Life Sci. 2017;74(16):2959-77. doi:10.1007/s00018-017-2509-x.

12. Marciano F, Vajro P. Oxidative stress and gut microbiota. In: Gracia-Sancho J, Salvadó J, editors. Gastrointestinal Tissue. Academic Press; 2017. pp. 113-23. doi:10.1016/B978-0-12-8053775.00008-4.

13. López P, Sánchez B, Margolles A, Suárez A. Intestinal dysbiosis in systemic lupus erythematosus: Cause or consequence? Curr Opin Rheumatol. 2016;28(5):515-22.

doi:10.1097/BOR.0000000000000309. 
14. Jeon SW, Kim Y. Inflammation-induced depression: Its pathophysiology and therapeutic implications. J Neuroimmunol. 2017;313:92-8;. doi:10.1016/j.jneuroim.2017.10.016.

15. Alekseenko AV, Kolos VA, Waseem TV, Fedorovich SV. Glutamate induces formation of free radicals in rat brain synaptosomes. Biophysics. 2009;54:617-20. doi:10.1134/S000635090905011X.

16. Sudo N, Microbiome. HPA axis and production of endocrine hormones in the gut. Adv Exp Med Biol. 2014;817:177-94. doi:10.1007/978-1-4939-0897-4_8.

17. Bai R, Liu S, Zhao Y, Cheng Y, Li S, Lai A, et al. Depressive and anxiety disorders in systemic lupus erythematosus patients without major neuropsychiatric manifestations. J Immunol Res. 2016; 2016:2829018; doi:10.1155/2016/2829018.

18. Gorwood P. Neurobiological mechanisms of anhedonia. Dialogues Clin Neurosci. 2008;10(3):291-9. doi:10.31887/DCNS.2008.10.3/pgorwood.

19. Stroebe M, Schut $H$, Boerner K. Cautioning health-care professionals: Bereaved persons are misguided through the stages of grief. Omega (Westport). 2017;74(4):455-73. doi:10.1177/0030222817691870.

20. Hevia A, Milani C, López P, Cuervo A, Arboleya S, Duranti S, et al. Intestinal dysbiosis associated with systemic lupus erythematosus. MBio. 2014;5(5):e01548-14. doi:10.1128/mBio.01548-14.

21. Furness JB, Rivera LR, Cho HJ, Bravo DM, Callaghan B. The gut as a sensory organ. Nat Rev Gastroenterol Hepatol. 2013;10(12):729-40. doi:10.1038/nrgastro.2013.180.

22. Zechner EL. Inflammatory disease caused by intestinal pathobionts. Curr Opin Microbiol. 2017;35:64-9. doi:10.1016/j.mib.2017.01.011.

23. Zhao Y, Lukiw WJ. Bacteroidetes neurotoxins and inflammatory neurodegeneration. Mol Neurobiol. 2018;55(12):9100-7. doi:10.1007/s12035-018-1015-y.

24. Wexler HM. Bacteroides. The good, the bad, and the nitty-gritty. Clin Microbiol Rev. 2007;20(4):593621. doi:10.1128/CMR.00008-07.

25. Gibiino G, Lopetuso LR, Scaldaferri F, Rizzatti G, Binda C, Gasbarrini A. Exploring Bacteroidetes: Metabolic key points and immunological tricks of our gut commensals. Dig Liver Dis. 2018;50(7):635-9. doi:10.1016/j.dld.2018.03.016.

26. Reese AT, Dunn RR. Drivers of microbiome biodiversity: A review of general rules, feces, and ignorance. mBio. 2018;9(4):e01294-18. doi:10.1128/mBio.01294-18.

27. Mosca A, Leclerc M, Hugot JP. Gut microbiota diversity and human diseases: Should we reintroduce key predators in our ecosystem? Front Microbiol. 2016;7:455. doi:10.3389/fmicb.2016.00455.

28. Jiang H, Ling Z, Zhang Y, Mao H, Ma Z, Yin Y, et al. Altered fecal microbiota composition in patients with major depressive disorder. Brain Behav Immun. 2015;48:186-94;. doi:10.1016/j.bbi.2015.03.016.

29. Brown K, DeCoffe D, Molcan E, Gibson DL. Diet-induced dysbiosis of the intestinal microbiota and the effects on immunity and disease. Nutrients. 2012;4(8):1095-119. doi:10.3390/nu4081095. 
Figures

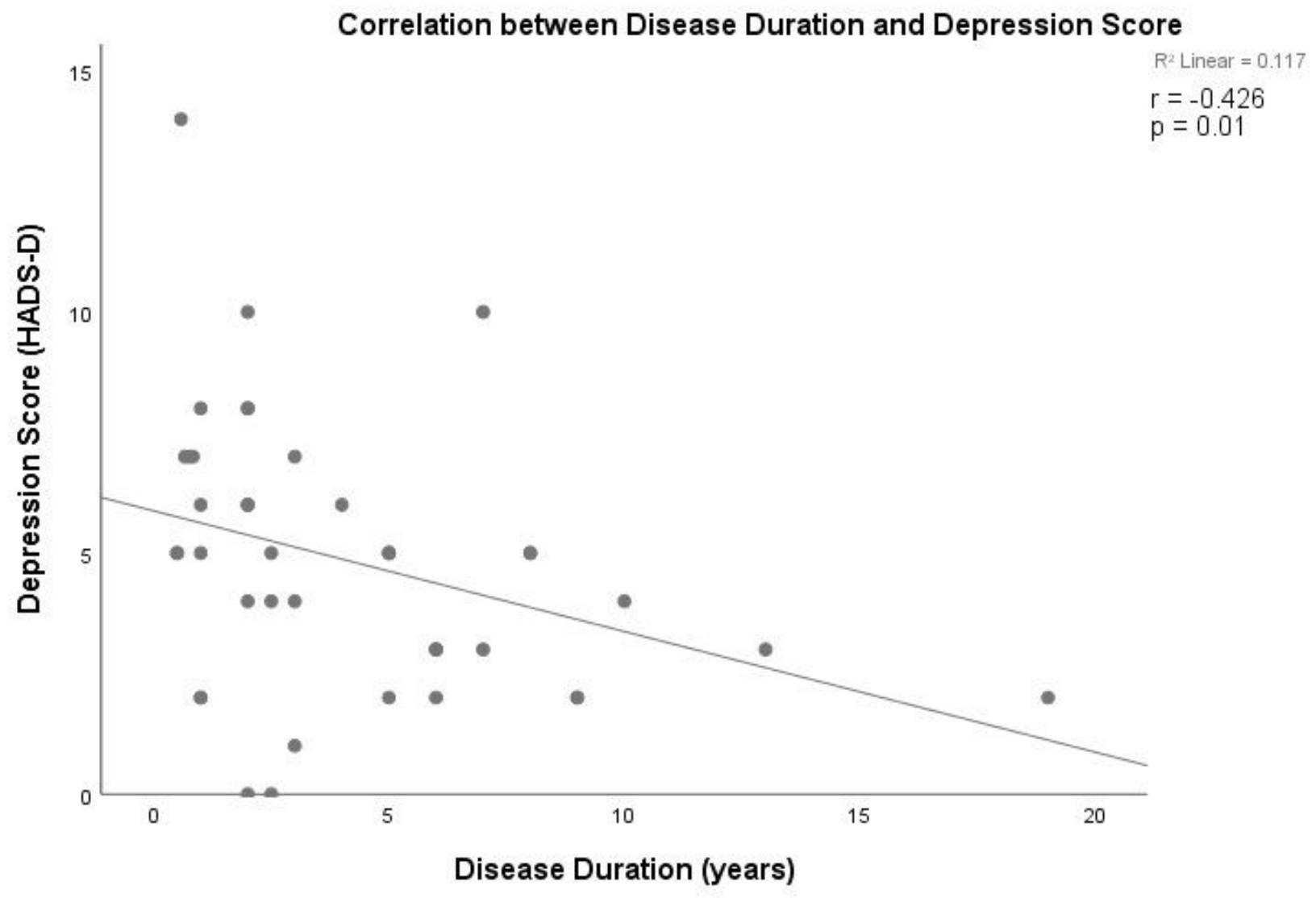

Figure 1

Correlation between disease duration and depression score 


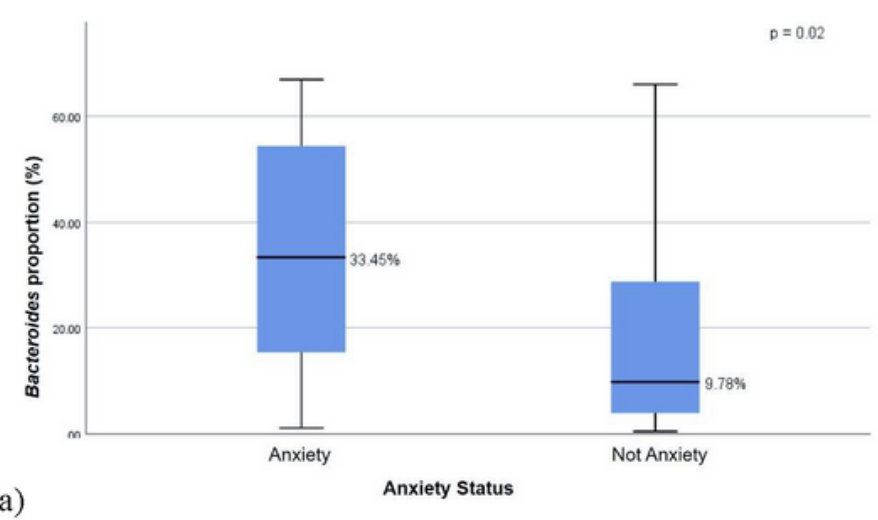

(a)

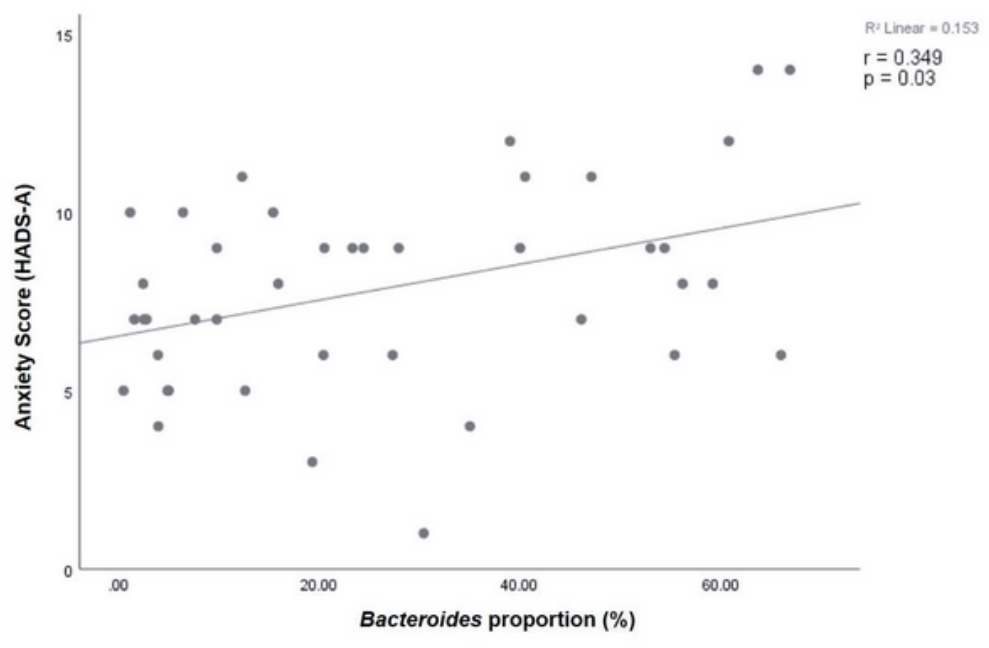

(b)

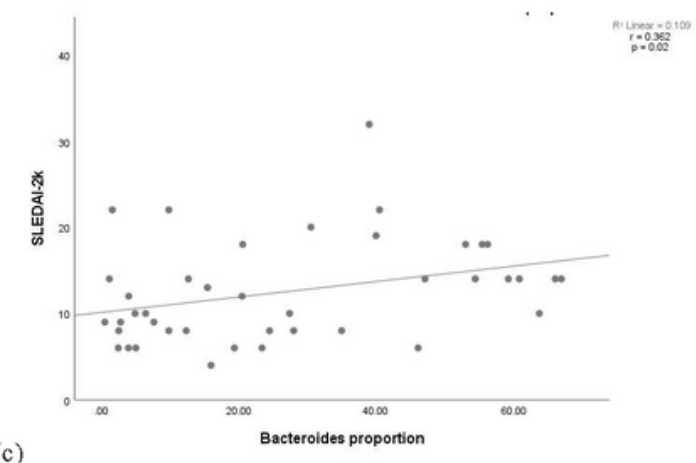

\section{Figure 2}

Bacteroides in SLE patients (a) according to anxiety status (b) correlation with anxiety score and (c) correlation with SLE disease activity score. 


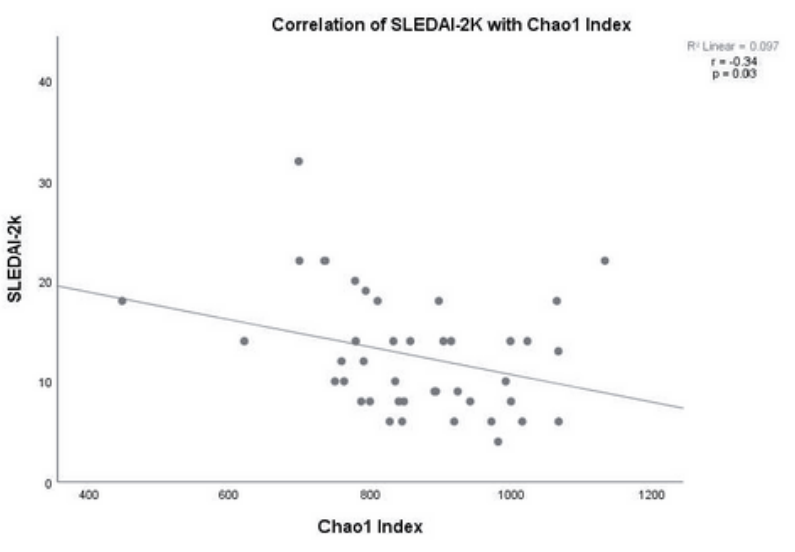

(a)

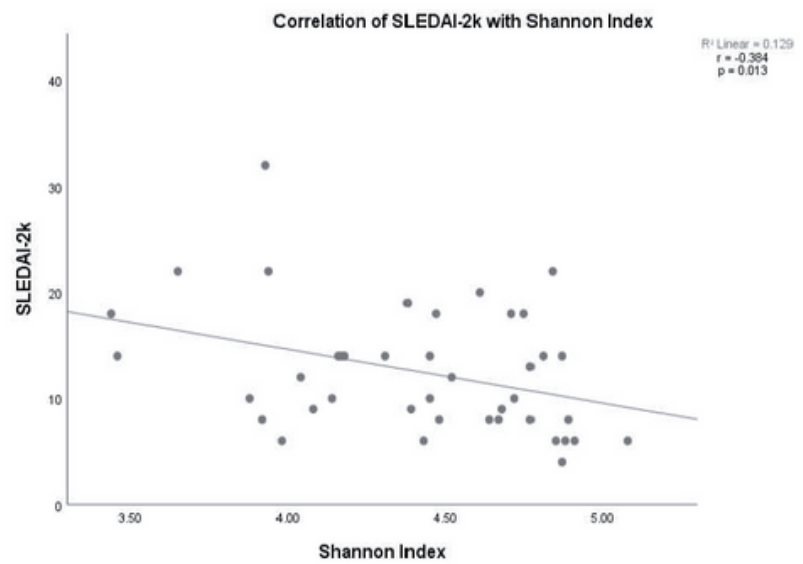

(b)

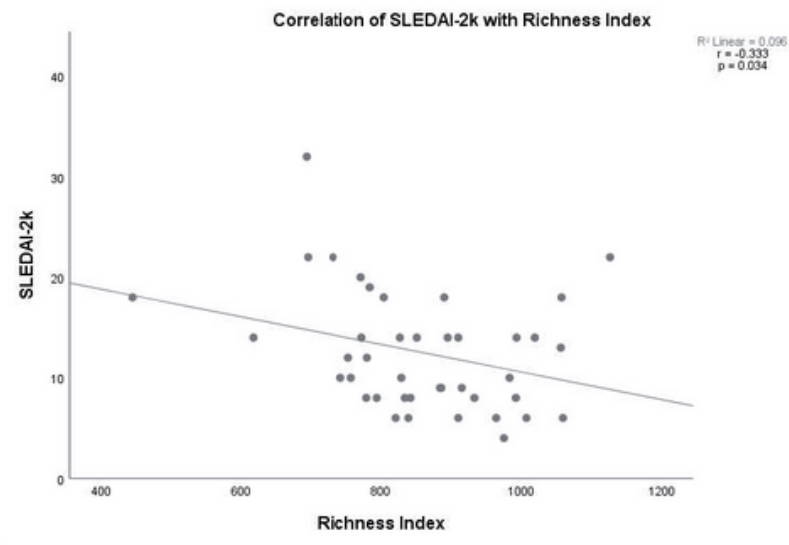

\section{Figure 3}

Correlation of diversity indices: (a) Chao1 index, (b) Shannon index, and (c) Richness index with SLE disease activity (SLEDAI-2K). 